\title{
Health care resource utilization and cost of severe hypoglycemia treatment in insulin-treated patients with diabetes in the United States
}

\author{
Sanjay Bajpai, PhD; Siew Wong-Jacobson; Dongju Liu, MS; Beth Mitchell, MPH, BSN, RN; Ginger Haynes, PhD; \\ Kristen Syring, PhD; Ayad K Ali, PhD; and Chanadda Chinthammit, BSPharm, MS, PhD
}

\section{What is already known about this subject}

- Treatment of severe hypoglycemia can involve health care resource utilization (HCRU), which contributes to the cost associated with the management of diabetes.

- It is challenging to determine the precise incidence, prevalence, HCRU, and costs by payer type that can accompany these events.

\section{What this study adds}

- This study provides a systematic analysis of the patient profiles of those who experience severe hypoglycemia that involve HCRU by different payer types.

- This novel analysis assigns cost to each resource rather than describing the total cost for the severe hypoglycemic event.

- Ultimately, these data provide value to payers and clinicians to make more informed decisions about HCRU when treating severe hypoglycemia.

\section{Author affiliations}

Sanjay Bajpai, PhD; Siew Wong-Jacobson; Dongju Liu, MS; Beth Mitchell, MPH, BSN, RN; Ginger Haynes, PhD; Kristen Syring, PhD; Ayad K. Ali, PhD; and Chanadda Chinthammit, BSPharm, MS, PhD, Eli Lilly and Company, Indianapolis, IN.

AUTHOR CORRESPONDENCE:

Sanjay Bajpai, 317.276.1798;

sanjay.bajpai@lilly.com

J Manag Care Spec Pharm 2021;27(3):385-91

Copyright $\odot 2021$, Academy of Managed Care Pharmacy. All rights reserved.

\section{ABSTRACT}

BACKGROUND: Hypoglycemia is a major limiting factor in achieving glycemic control in persons with diabetes. In some instances, recovery from a severe hypoglycemia event may require health care resource utilization (HCRU), including the use of emergency medical services (EMS), visits to the emergency department (ED), and inpatient hospitalization.

OBJECTIVES: To (a) describe the profiles of patients who experience severe hypoglycemic events and (b) characterize HCRU and the associated cost related to severe hypoglycemia treatment.
METHODS: This retrospective, observational cohort study used administrative claims data from IBM MarketScan Research Databases. The study examined a cohort of subjects who experienced severe hypoglycemic events that involved HCRU during the 1-year index period. Baseline patient demographic data were collected according to patient profiles, such as payer type, type of diabetes, age, and type of insulin. HCRU and the associated cost data categorized by the patient profiles and care progression scenarios were described.

RESULTS: 9,563 patients from the IBM MarketScan Research Databases experienced a severe hypoglycemic event during the index period and were included in the study; approximately $75 \%$ of those patients did not experience a severe hypoglycemic event in the previous year. Of the 9,563 patients in the cohort, the largest patient profile $(n=1,767,18.5 \%)$ consisted of those who were on Medicaid, had type 2 diabetes, and used basal/bolus or premixed-only insulins. Overall, more than $90 \%$ of the index severe hypoglycemic events involved visits to the ED. EMS claims in the 24 hours before the ED visit were found for half of the severe hypoglycemic events (51.5\%).

CONCLUSIONS: Differences in HCRU and the associated costs for the treatment of severe hypoglycemia were observed among patients based on insurance, diabetes, and insulin types. Clinicians need to be aware 
of these differences. Optimizing treatment of severe hypoglycemia, specifically EMS care, and examining patient profiles to develop targeted interventions could potentially provide benefits to patients and reduce cost and resource utilization.

Hypoglycemia, or low blood glucose, is a major limiting factor in achieving glycemic control in persons with diabetes (PWD), especially those on insulin therapy. Severe, or level 3, hypoglycemia is a low blood glucose event characterized by an altered mental or physical state and requiring assistance from another person to recover. ${ }^{1}$ Left untreated, severe hypoglycemia can lead to loss of consciousness, seizure, coma, or death.

For some severe hypoglycemic events, treatment may involve health care resource utilization (HCRU), which can include the use of emergency medical services (EMS), visits to the emergency department (ED), and inpatient hospitalization. A previous analysis using data from 15 clinical trials that assessed HCRU for treatment of severe hypoglycemia found that nearly $30 \%$ of severe hypoglycemic events that occurred during the clinical trials required an ambulance or emergency services, and approximately $18 \%$ used the ED or required hospital stays. ${ }^{2}$

The severity of the hypoglycemic event is associated with increased used of health care resources and the associated costs. ${ }^{3}$ These costs add to the significant financial burden placed on the PWD and the health care system. It was previously estimated that the total cost of diabetes in the United States in 2017 was $\$ 327$ billion. Of this total cost, \$237 billion (72.5\%) was a result of direct medical costs, and $\$ 90$ billion (27.5\%) was because of reduced productivity. ${ }^{4}$ Severe hypoglycemic events can account for a portion of that cost. ${ }^{5}$ The cost associated with HCRU for the treatment of severe hypoglycemia can provide valuable information on the financial burden that these events place on the health care system.

The purpose of this cohort study was to describe costs associated with severe hypoglycemic events among patients with type 1 (T1D) or type 2 diabetes mellitus (T2D). Furthermore, this study described the incidence and prevalence of severe hypoglycemic events that involved HCRU and the profiles of patients that experienced these events. HCRU and the associated cost, specifically the gross payment from insurer to the provider, related to severe hypoglycemia treatment were characterized using administrative claims data. Importantly, this study describes the overall population and also breaks down the data by payer type, diabetes type, type of insulin used, and care progression scenarios, allowing for an in-depth understanding of the use of HCRU and associated costs related to severe hypoglycemia.

\section{Methods}

\section{STUDY DESIGN}

This retrospective, observational cohort study used administrative claims data from the IBM MarketScan Commercial, Medicare Supplemental, and Multi-State Medicaid Databases from December 2015 to December 2017. The MarketScan Research Databases contain individual-level and deidentified health care claims information from employers, health plans, hospitals, and Medicare and Medicaid programs. The data were used to assess the incidence and prevalence of severe hypoglycemic events in the United States.

In this study, severe hypoglycemic events were identified using (a) the International Classification of Diseases, Ninth Revision, Clinical Modification (ICD-9-CM) coding algorithm validated by Ginde et al. (2008) ${ }^{6}$; (b) International Classification of Diseases, Tenth Revision, Clinical Modification (ICD-CM-10) codes for hypoglycemia; and (c) claims data reflecting the use of EMS or ED. ${ }^{6,7}$ According to the American Diabetes Association, hypoglycemia that requires a third party to recover is considered a severe hypoglycemic event. Because all events captured in this database involved EMS or ED intervention, they were considered severe hypoglycemic events for this analysis.

The index event was defined as the first severe hypoglycemic event between December 1, 2016, and November 30,2017 , the prespecified index period. The year before the index period, December 1, 2015-November 30, 2016, was used to determine if the participant experienced a severe hypoglycemic event before the index event. From June 1, 2016, to November 30,2017 , claims data were analyzed to ensure that participants received an insulin prescription.

The outcome measures (HCRU and associated costs) were summarized by patient profiles, such as insurance payer type, type of diabetes, and type of insulin, as well as the 12 possible care progression scenarios involving at least one of the following: EMS, ED, observation case visits, and inpatient hospitalization (Supplementary Table 1, available in online article). HCRU and costs were considered related to the index event if the HCRU encounter occurred within 24 hours of the previous HCRU encounter in the care progression scenario.

\section{STUDY POPULATION}

This study examined patients who experienced a severe hypoglycemic event during the index period. Patients who 
met all the following criteria were included in the study: (a) filled at least 1 script of insulin between June 1, 2016, and November 30, 2017; (b) had at least 2 diagnoses of T1D or T2D (ICD-9-CM: 250.xx, ICD-10-CM: E10.xx, E11.xx) or 1 diagnosis of T1D or T2D and 1 antidiabetic medication within 12 months, between June 1, 2016, and November 30, 2017; (c) no presence of gestational diabetes (ICD-9-CM: 648.8x, ICD-10-CM: O24.4x), diabetes mellitus complicating pregnancy, childbirth or the puerperium (ICD-9-CM: 648.0x, ICD-10-CM: O24.xx), nonclinical diabetes (ICD-9-CM: 790.29, ICD-10-CM: R73.09), and secondary diabetes (ICD-9-CM: 249.x, ICD-10-CM: E09.xx,E08.xx) between June 1, 2016, to Nov 30, 2017; (d) met the claims-based algorithm to identify T1D or T2D between December 1, 2015, and November 30, 20178; (e) maintained continuous medical and pharmacy enrollment from June 1, 2016, to December 31, 2017; and (f) experienced severe hypoglycemia between December 1, 2016, and November 30, 2017.

The study time period is described in Supplementary Figure 1 (available in online article).

\section{INDEPENDENT AND OUTCOME VARIABLES}

Patient demographic data were collected, including payer type (Medicare, commercial, and Medicaid); type of diabetes ascertained using a modified algorithm adapted from Klompas et al. $(2013)^{8}$; age (pediatric [aged < 18 years] vs. adult [aged $\geq 18$ years]); and type of insulin (basal only, basal/bolus, and premixed only). For the T1D population, the sample size was too small to divide among the 3 insulin types. Therefore, during the analysis, patients on basal-only, basal/bolus, and premixed-only insulin were combined and described as "any insulin." For the T2D population, patients using basal/bolus and premixed-only insulin were combined and described as "other." Those using basal-only insulin were kept separate for the T2D analysis. HCRU and the associated cost data, specifically the gross payment from the insurer to the provider, categorized by the patient profiles and care progression scenarios, were described. The care progression scenarios are presented in Supplementary Table 1 (available in online article.

\section{INCIDENCE AND PREVALENCE}

The incidence of severe hypoglycemia was defined as the proportion or rate of persons who develop a condition during a particular time period. In this study, incidence was calculated as the number of patients who experienced a severe hypoglycemic event during the index period without an event in the previous year compared with all patients with T1D or T2D who had an insulin claim but no severe hypoglycemic event between December 1, 2015, and November 30, 2017.
The prevalence of severe hypoglycemia was calculated as the ratio of the number of patients who experienced a severe hypoglycemic event during the index period to all patients with T1D or T2D who had an insulin claim between June 1, 2016, and November 30, 2017.

\section{STATISTICAL ANALYSES}

Summary statistics were used to describe demographics, HCRU, and associated cost of care progression scenario data. The median cost and corresponding interquartile range (25th percentile-75th percentile) for the index severe hypoglycemic event were calculated. Inferential statistics (including test of significance or regression analyses) were not conducted because sample sizes were not large enough to provide robust analysis. SAS EG was used for the statistical summary analysis (SAS Institute, Cary, NC).

\section{Results}

\section{PREVALENCE AND INCIDENCE OF SEVERE HYPOGLYCEMIA}

The prevalence of PWD included in this study who experienced a severe hypoglycemic event between December 1 , 2016, and November 30, 2017, was 2.8\% (9,653/335,766; data not shown). The incidence of severe hypoglycemic events during the same time period, calculated among patients who did not experience severe hypoglycemia the previous year, was $2.4 \%$ (7,223/297,118; data not shown).

\section{PATIENT DEMOGRAPHICS}

Of the 335,766 patients, 9,563 experienced a severe hypoglycemic event during the index period (Supplementary Figure 2, available in online article), and approximately $75 \%$ of those patients did not experience a severe hypoglycemic event in the 12 months before the event. The average patient age was 54 years (Table 1). Approximately $93 \%$ of the patients were adults, and $52.7 \%(n=5,036)$ were female. In this study, patients were more likely to have T2D (62.3\%) than T1D (37.7\%). Commercial, Medicare, and Medicaid insurance were represented by similar proportions. The most commonly used insulin therapy was basal/bolus insulin (79.2\%).

\section{SEVERE HYPOGLYCEMIA PATIENT PROFILES}

Of the 9,563 patients who experienced a severe hypoglycemic event during the index period, the largest patient profile $(n=1,767,18.5 \%)$ consisted of those who had Medicaid, had T2D, and used basal/bolus or premixed-only insulins. Patients with T1D with commercial insurance treated with any type of insulin therapy also accounted for a large proportion $(n=1,732,18.1 \%)$. The patient profiles are shown in Supplementary Figure 3 (available in online article). 


\begin{tabular}{|c|c|c|}
\hline & \multicolumn{2}{|c|}{ Prevalence } \\
\hline & $\mathbf{n}$ & $\%$ \\
\hline Total patients in the cohort & 9,563 & 100.0 \\
\hline \multicolumn{3}{|l|}{ Age at index } \\
\hline Mean (SD) & \multicolumn{2}{|c|}{$54(20)$} \\
\hline Pediatrics (aged < 18 years) & 630 & 6.6 \\
\hline Adult (aged $\geq 18$ years) & 8,933 & 93.4 \\
\hline \multicolumn{3}{|l|}{ Gender } \\
\hline Female & 5,036 & 52.7 \\
\hline Male & 4,527 & 47.3 \\
\hline \multicolumn{3}{|l|}{ Geographical region } \\
\hline North Central & 1,863 & 19.5 \\
\hline North East & 1,032 & 10.8 \\
\hline South & 2,409 & 25.2 \\
\hline West & 514 & 5.4 \\
\hline Unknown/missing & 3,745 & 39.2 \\
\hline \multicolumn{3}{|l|}{ Insurance payer plan } \\
\hline Commercial & 3,392 & 35.5 \\
\hline Medicare & 2,442 & 25.5 \\
\hline Medicaid & 3,729 & 39.0 \\
\hline \multicolumn{3}{|l|}{ Diabetes type } \\
\hline Type 1 diabetes & 3,602 & 37.7 \\
\hline Type 2 diabetes & 5,961 & 62.3 \\
\hline \multicolumn{3}{|l|}{ Insulin type } \\
\hline Basal only & 1,529 & 16.0 \\
\hline Basal/bolus & 7,571 & 79.2 \\
\hline Premixed only & 463 & 4.8 \\
\hline
\end{tabular}

\section{HEALTH CARE PROGRESSION SCENARIOS}

The most common health care progression scenarios used by patients were scenarios number 4 and 8 (Supplementary Table 1, available in online article). Scenario 4 involved EMS and an ED visit without an observation period, and scenario 8 involved the patient going directly to the ED and being released without observation. These data are presented in Supplementary Table 2 (available in online article).

Overall, over $90 \%$ of the index severe hypoglycemic events involved visits to the ED (Figure 1). EMS was called during approximately half of the events (51.5\%), whereas during $48.5 \%$ of events, the PWD were taken directly to the ED without EMS. During severe hypoglycemic events that involved EMS, most PWD were transported to the
ED (83.4\%). Of all PWD that visited the ED, 32.7\% were hospitalized. Specifically, before hospitalization, nearly $18 \%$ of PWD that visited the ED went directly to ED, and $15 \%$ were transported by EMS. HCRU was similar for the 3 different payer types (Supplementary Figure 4, available in online article).

After stratification by insurance payer type, PWD who went directly to the ED accounted for the largest proportion among the commercially insured and Medicaid subgroups (36.0\% and $34.7 \%$, respectively). On the other hand, PWD who called for EMS and were later transported to the ED accounted for the largest proportion among the Medicare subgroup. The Medicare subgroup demonstrated a greater use of all 3 health care services (EMS, ED, and hospitalization) but with lower numbers for "ED only."

\section{HCRU COST ASSOCIATED WITH SEVERE HYPOGLYCEMIA TREATMENT}

The median cost associated with HCRU for the treatment of severe hypoglycemia were described for each care progression scenario (Supplementary Table 2 and Supplementary Figure 5, available in online article). While EMS treatment alone had the lowest associated median cost (25th-75th [interquartile range]) per event (\$140 [\$49.5-\$410.4]), the median cost of hospitalization after EMS treatment alone was associated with the highest median cost per event (\$11,988.8 [\$3728.9-\$33192.2]). When analyzed by payer type, managed Medicaid was associated with the lowest HCRU costs, followed by Medicare and commercial payers (Supplementary Figure 6, available in online article).

\section{Discussion}

This retrospective, observational study described the profiles of PWD who experienced severe hypoglycemia, as well as the HCRU and associated costs of severe hypoglycemia treatment. This study adds to the limited number of descriptive studies that have examined the cost associated with severe hypoglycemia treatment. Unique to this study, HCRU is described using carefully defined care progression scenarios, which provide a more in-depth portrayal of how health care resources are being used and which combinations of resources are most common. Furthermore, the study design enables descriptions of HCRU and costs based on payer type at a national level.

The claims data from this study indicate that most severe hypoglycemic events requiring HCRU involve ED visits, with or without previous EMS treatment. However, approximately half of the PWD included in this study were first treated by EMS. While the present data indicate that less than $10 \%$ of the study population encountered EMS 


\section{FIGURE 1 HCRU Care Progression Scenarios}

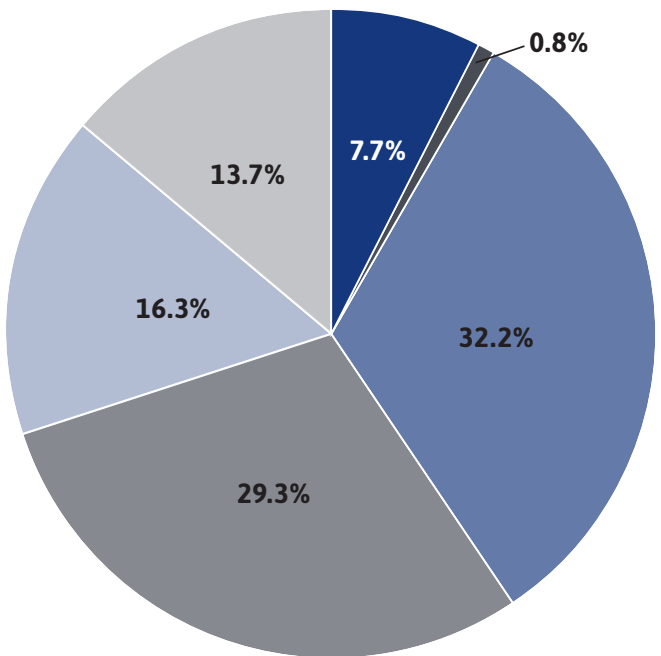

EMS + ED

ED + hospitalization EMS + ED + hospitalization

Note: This figure presents the percentage of total participants that used each HCRU care progression scenario.

$E D=$ emergency department; $E M S=$ emergency medical services; $H C R U$ = health care resource utilization.

alone, a previous study using the National Emergency Medical Services Information System (NEMSIS), a research database, reported that over $40 \%$ of patients who were treated by EMS for hypoglycemia were released and did not have further HCRU encounters. ${ }^{9}$ While the percentage of PWD using each care progression scenario is fairly similar among the payer types, commercial payers covering PWD have the highest costs, followed by Medicare and Medicaid. These cost differences are likely partially driven by lower negotiated hospitalization costs. ${ }^{10}$

To fully capture the burden that severe hypoglycemic events place on PWD and the health care system, the incidence and prevalence of severe hypoglycemic events needs to be accurately measured. Administrative claims data allow for an estimation of the incidence and prevalence of severe hypoglycemia. Overall, the incidence and prevalence of severe hypoglycemia in this study were $2.4 \%$ and $2.8 \%$, respectively. Similarly, previous studies demonstrated that the incidence of severe hypoglycemia requiring HCRU was between $1.4 \%$ and $1.5 \%,{ }^{11,12}$ while the prevalence was between $1.1 \%$ and $4.2 \% .^{13-15}$

However, a precise measure of incidence and prevalence has proven difficult for 2 reasons. First, the definition of severe hypoglycemia requires only that the blood glucose is low enough to require assistance from a third party. This definition may lead to the inclusion of nonsevere events and exclusion of severe events from analyses. Second, PWD do not report all the severe hypoglycemic events that they experience to health care providers. Therefore, capturing all severe hypoglycemic events is not likely. While not all severe hypoglycemic events require HCRU, this study design provides a systematic measurement to consistently capture the events within the inclusion criteria.

In fact, while the current study requires HCRU for the treatment of severe hypoglycemia, previous studies indicate that most events occur at home and do not involve any HCRU. Specifically, in the U.S. cohort of the global Conversations and Reactions Around Severe Hypoglycemia (CRASH) study, over $77 \%$ of participants with T1D and $81 \%$ of participants with $\mathrm{T} 2 \mathrm{D}$ reported that their most recent severe hypoglycemic event occurred at home. ${ }^{16}$ Furthermore, only about $10 \%$ of the participants reported calling EMS or going to the ED, indicating that the data included in the present study, which relies on claims data, cannot account for all severe hypoglycemic events.

Regardless of HCRU, severe hypoglycemia can have profound psychological and social effects on PWD. It is also important to prepare and protect PWD, specifically by increasing education regarding the prevention and management of hypoglycemic events (for PWD, caregivers, parents, teachers, and the community as a whole) by identifying people at greatest risk and improving access to medications for the treatment of severe hypoglycemia (i.e., a glucagon device that is easy to deliver a full rescue dose to possibly avoid emergency HCRU altogether).

The population in this study included persons with T1D or T2D. Persons with T1D are more likely to experience a severe hypoglycemic event ${ }^{17}$; however, because persons with T2D account for approximately $90 \%-95 \%$ of all cases of diabetes mellitus, ${ }^{18}$ a larger proportion of patients included in this study have T2D. Therefore, these results indicate that those experiencing severe hypoglycemia that requires HCRU are most often persons with T2D using premixed or basal/bolus insulin, which is a more intensive treatment than basal-only insulin.

The broad inclusion criteria of this study allow for the results to be generalized to a large population. The type of diabetes, age, or payer type were not exclusionary. Further, the dataset is extensive and describes patient profiles, HCRU, and associated costs at the national level, including more than 1 payer type, but also enables the data to be broken down and examined multiple ways. This study provides 
a detailed view of patients who use insulin and their severe hypoglycemic events.

\section{LIMITATIONS}

Although this study involves a large dataset, there are several limitations. To be included in this study, PWD needed to be on insulin and have insurance, meaning the study population was not representative of all PWD.

Only hypoglycemic events that involved HCRU were included in the study. Not all severe hypoglycemic events require HCRU, and events that involve the ED or EMS are assumed to be severe, but this cannot be known based on claims data alone. Also, administrative claims data cannot account for these events. Therefore, this study was not able to quantify the incidence and prevalence of all severe hypoglycemic events. Furthermore, coding errors in administrative claims data cannot be accounted for, and charges for HCRU are not necessarily consistent. For example, EMS treatment does not always result in a charge to the insurance company.

Finally, as with any data source, MarketScan data have limitations, some due to the nature of the MarketScan sample population. One such limitation is that the database is a large sample, but because the sample is not random, it may contain biases or fail to generalize well to other populations or to those without health insurance coverage. However, these data can complement other datasets or be used as benchmarks against them. Also, the data come mostly from large employers, so medium and small firms may be underrepresented, although MarketScan databases include a large amount of data contributed from a variety of health plans.

\section{CONCLUSIONS}

Optimizing treatment of severe hypoglycemia could be a substantial cost-saving option for patients who experience these events. Examining the patient profiles and developing targeted intervention based on patient characteristics to prevent or better treat severe hypoglycemia could provide benefits to patients. Furthermore, since EMS was the first HCRU encounter for approximately half of all PWD in this study, valuing EMS care to ultimately reduce the proportion of PWD requiring transport to ED or hospitalization could potentially provide benefits and reduce the financial burden of severe hypoglycemia.

\section{DATA-SHARING STATEMENT}

The datasets analyzed during the current study are available from the corresponding author on reasonable request.

\section{DISCLOSURES}

This study was funded by Eli Lilly and Company. All authors are employees and shareholders of Eli Lilly and Company.

The data presented here have been presented in poster form at AMCP Nexus 2020 Virtual, October 19-23, 2020; ADCES Virtual Conference 2020, August 13-16, 2020; and Virtual ISPOR 2020, May 18-20, 2020 .

\section{REFERENCES}

1. American Diabetes Association. 6. Glycemic targets: standards of medical care in diabetes-2020. Diabetes Care. 2020;43(Suppl 1):S66-S76.

2. Heller SR, Frier BM, Herslov ML, Gundgaard J, Gough SC. Severe hypoglycaemia in adults with insulin-treated diabetes: impact on healthcare resources. Diabet Med. 2016;33(4):471-77.
3. Pawaskar M, Iglay K, Witt EA, Engel SS, Rajpathak S. Impact of the severity of hypoglycemia on health-related quality of life, productivity, resource use, and costs among U.S. patients with type 2 diabetes. J Diabetes Complications. 2018;32(5):451-57.

4. American Diabetes Association. Economic costs of diabetes in the U.S. in 2017. Diabetes Care. 2018;41(5):917-28.

5. Bajpai S, Poehlmann J, Mitchell B, Hankosky E, Valentine W. Economic impact of nasal glucagon for the treatment of severe hypoglycemia in patients with diabetes treated with insulin in the U.S. J Manag Care Spec Pharm. 2020; 26(4-a):S28 [abstract E9]. Accessed February 16, 2021. https://doi. org/10.18553/jmcp.2020.26.4-a.s1

6. Ginde AA, Blanc PG, Lieberman RM, Camargo CA Jr. Validation of ICD-9-CM coding algorithm for improved identification of hypoglycemia visits. BMC Endocr Disord. 2008;8:4.

7. Karter AJ, Warton EM, Moffet HH, et al. Revalidation of the hypoglycemia risk stratification tool using ICD-10 codes. Diabetes Care. 2019;42(4):e58-e59.

8. Klompas M, Eggleston E, McVetta J, Lazarus R, Li L, Platt R. Automated detection and classification of type 1 versus type 2 diabetes using electronic health record data. Diabetes Care. 2013;36(4):914-21.

9. Kaufmann MA, Nelson DR, Kaushik P, Mann NC, Mitchell B. Hypoglycemia emergencies: factors associated with prehospital care, transportation status, emergency department disposition, and cost. Prehosp Emerg Care. 2019;23(4):453-64.

10. Woodworth L, Romano PS, Holmes JF. Does insurance status influence a patient's hospital charge? Appl Health Econ Health Policy. 2017;15(3):353-62.

11. Pathak RD, Schroeder EB, Seaquist ER, et al. Severe hypoglycemia requiring medical intervention in a large cohort of adults with diabetes receiving care in u.s. integrated health care delivery systems: 2005-2011. Diabetes Care. 2016;39(3):363-70. 
12. Wang J, Geiss LS, Williams DE, Gregg EW. Trends in emergency department visit rates for hypoglycemia and hyperglycemic crisis among adults with diabetes, United States, 2006-2011. PLoS One. 2015;10(8):e0134917.

13. Liu J, Wang R, Ganz ML, Paprocki Y, Schneider D, Weatherall J. The burden of severe hypoglycemia in type 1 diabetes. Curr Med Res Opin. 2018;34(1):171-77.

14. Liu J, Wang R, Ganz ML, Paprocki Y, Schneider D, Weatherall J. The burden of severe hypoglycemia in type 2 diabetes. Curr Med Res Opin. 2018;34(1):179-86.
15. Quilliam B, Simeone J, Ozbay A, Kogut S. The incidence and costs of hypoglycemia in type 2 diabetes. Am J Manag Care. 2011;17(10):673-80.

16. Snoek FJ, Jiletcovici J, Bushnell DM III, et al. 285-OR: Conversations and reactions around severe hypoglycemia (CRASH): U.S. results from a global survey of people with T1DM or insulin-treated T2DM and caregivers [abstract]. Diabetes. 2019;68(Suppl 1). Accessed February 16, 2021. https://doi.org/10.2337/ db20-268-OR
17. Briscoe VJ, Davis SN. Hypoglycemia in type 1 and type 2 diabetes: physiology, pathophysiology, and management. Clinical Diabetes. 2006;24(3):115-21.

18. Zimmet P, Alberti KG, Shaw J. Global and societal implications of the diabetes epidemic. Nature. 2001; 414(6865):782-87. 